\title{
Rapid assessment of the stability of DNA duplexes by impedimetric real-time monitoring of chemically induced denaturation $\dagger$
}

\author{
B. van Grinsven, ${ }^{* a}$ N. Vanden Bon, ${ }^{b}$ L. Grieten, ${ }^{a}$ M. Murib, ${ }^{a}$ S. D. Janssens, ${ }^{a}$ K. Haenen, ${ }^{a c}$ E. Schneider, ${ }^{d}$ \\ S. Ingebrandt, ${ }^{d}$ M. J. Schöning, ${ }^{e}$ V. Vermeeren, ${ }^{b}$ M. Ameloot, ${ }^{b}$ L. Michiels, ${ }^{b}$ R. Thoelen, ${ }^{a f}$ W. De Ceuninck ${ }^{a c}$ \\ and P. Wagner ${ }^{a c}$
}

\section{Received 12th January 2011, Accepted 3rd March 2011}

DOI: 10.1039/c1lc20027e

In this article, we report on the electronic monitoring of DNA denaturation by $\mathrm{NaOH}$ using electrochemical impedance spectroscopy in combination with fluorescence imaging as a reference technique. The probe DNA consisting of a 36-mer fragment was covalently immobilized on nanocrystalline-diamond electrodes and hybridized with different types of 29-mer target DNA (complementary, single-nucleotide defects at two different positions, and a non-complementary random sequence). The mathematical separation of the impedimetric signals into the time constant for $\mathrm{NaOH}$ exposure and the intrinsic denaturation-time constants gives clear evidence that the denaturation times reflect the intrinsic stability of the DNA duplexes. The intrinsic time constants correlate with calculated DNA-melting temperatures. The impedimetric method requires minimal instrumentation, is label-free and fast with a typical time scale of minutes and is highly reproducible. The sensor electrodes can be used repetitively. These elements suggest that the monitoring of chemically induced denaturation at room temperature is an interesting approach to measure DNA duplex stability as an alternative to thermal denaturation at elevated temperatures, used in DNA-melting experiments and single nucleotide polymorphism (SNP) analysis.

\section{A. Introduction}

One of the central challenges in human genomics is the detection and identification of single-nucleotide polymorphisms. Miniaturized assays such as microarrays play here an important role to allow for a massively parallelized readout in combination with small sample volumes. ${ }^{1,2}$ Disadvantages are the long reaction times at the scale of at least 16 hours, the complete lack of dynamic information on the DNA binding kinetics, the need for fluorescent labelling of the target DNA, and the sophisticated optical readout techniques. Also, microarrays are in principle

${ }^{a}$ Hasselt University, Institute for Materials Research, Wetenschapspark 1, B-3590 Diepenbeek, Belgium. E-mail: bart.vangrinsven@uhasselt.be

${ }^{b}$ Hasselt University, Biomedical Research Institute, Agoralaan, B-3590 Diepenbeek, Belgium

'IMEC vzw-Division IMOMEC, Wetenschapspark 1, B-3590 Diepenbeek, Belgium

${ }^{d}$ University of Applied Sciences Kaiserslautern, Department of Informatics and Microsystems Technology, Amerikastrasse 1, D-66482 Zweibrücken, Germany

'Aachen University of Applied Sciences, Institute for Nano- and Biotechnologies, Ginsterweg 1, D-52428 Jülich, Germany

${ }^{f} X I O S$ University College Limburg, Department of Electronic Engineering, Agoralaan Building H, B-3590 Diepenbeek, Belgium

$\uparrow$ Electronic supplementary information (ESI) available. See DOI: $10.1039 / \mathrm{c} 1 \mathrm{lc} 20027 \mathrm{e}$ limited to the detection of known mutations albeit there is a recent progress to exploit the thermodynamic aspects of probe DNA-target DNA recognition to identify SNPs even in the presence of wild-type DNA. ${ }^{3}$ Alternatively, mutation analysis can be performed using techniques that exploit the denaturation of double-stranded (ds) DNA rather than the hybridization process. The best known examples are real-time polymerase chain reaction (PCR) with associated melting curve analysis ${ }^{4}$ and denaturing gradient gel electrophoresis (DGGE). ${ }^{5,6}$ Both techniques rely on the fact that DNA duplexes containing a SNP are less stable than complementary duplexes, resulting in lower denaturation (melting) temperatures. Nevertheless, both techniques need expensive instrumentation. Real-time PCR requires the use of fluorescent labels and DGGE is not suitable for highthroughput analysis.

Due to the inherent complexity of microarrays and the established denaturation-based approaches, strong efforts are put into the development of label-free detection techniques based on electronic readout principles. One of these electronic routes is the direct sequencing of DNA fragments with solid-state or haemolysin nanopores, utilizing the current-blocking effect. ${ }^{7-9}$ Alternatively, the DNA switching method on gold electrodes proposed by Rant et al. ${ }^{10}$ allows for real-time monitoring of hybridization and denaturation with the possibility to distinguish between complementary and mismatched fragments. Although 
the method requires no fluorescent labelling of the target DNA, labels are involved on the probe DNA. A switching effect lies also at the basis of the E-DNA sensor, ${ }^{11}$ which employs an electrochemical redox reaction rather than fluorimetric detection and offers femtomolar detection limits. A method without any labelling and auxiliary chemistry is the solution-gate field effect transistor (FET) device with the probe DNA directly immobilized on the gate oxide. ${ }^{12-14}$ Real-time monitoring of hybridization is in principle possible and the FETs can discriminate between complementary and mismatched strands at least under ex situ conditions. The sensing effect of FETs is attributed to the intrinsic negative charge of ss- and dsDNA fragments and to a redistribution of ionic charges at the proximity of the gate insulator during hybridization. ${ }^{15}$ DNA hybridization sensors based on impedance spectroscopy have been established with screen-printed carbon electrodes, ${ }^{16}$ mixed self-assembled monolayers on gold electrodes using a redox system, conjugated polymers, and GaN nanowires. ${ }^{17}$

Despite all recent progress, the aforementioned electronic or opto-electronic methods for DNA sensing have in common that they suffer from at least two or more of the following drawbacks: (i) need for high-end instrumentation and incompatibility with upgrading towards high-throughput assays; (ii) need for additional chemicals such as fluorescent dyes or redox mediators; (iii) lack of sensor regeneration capacity; (iv) missing proof that the sensor response is intrinsic and unaffected by conductivity effects related to the temperature or ionic composition of the buffer liquids; (v) insufficient statistics to demonstrate the reproducibility; (vi) lack of dynamic information on the kinetics of hybridization or denaturation events.

In this work, we will address these challenges by combining synthetic diamond electrodes, equipped with covalently immobilized probe DNA, with label-free, dynamic impedance readout and a stringent control on temperatures and medium composition using a miniaturized flow cell. Hereby, we will focus on the denaturation kinetics. One may expect a faster splitting of mismatched duplexes as compared to complementary duplexes, when they are exposed to a denaturation agent. Mismatchrelated denaturation times have indeed been recently reported by Özkumur et al., who employed label-free, optical interferometry on spotted microarrays, while the denaturation was induced by reducing the ionic strength of the buffer medium. ${ }^{18}$ Besides the intrinsic analogy with melting experiments, denaturation experiments offer the advantage to study duplex ensembles that are close to or at thermal equilibrium prior to the denaturation step. This starting condition is not necessarily fulfilled in the hybrization-based microarrays, where artefacts can occur due to metastable DNA hybrid states formed between non-complementary strands. ${ }^{19}$

\section{B. Experimental}

\section{Design of the sensor cell and the impedimetric readout system}

We use impedance spectroscopy, because this technique is especially versatile and can be employed not only for DNA hybridization, but also for protein detection and enzymatic reactions as shown in the review by Katz and Willner. ${ }^{20}$ Moreover, impedimetric sensors are comparatively easy in fabrication since they are two-terminal devices without the need for specific semiconductor doping profiles or advanced encapsulation techniques. From our prior impedimetric studies, there is indeed evidence that this method is suitable for monitoring DNA hybridization and denaturation under relevant buffer solution and temperature conditions. ${ }^{21}$ To measure the effect of denaturation of different DNA targets a sensor setup is constructed as shown in Fig. 1. This experimental setup consists of a homemade impedance spectroscopy unit as described in ref. 22 and a syringe system coupled to a Perspex flow cell with an inner volume of $110 \mu$ l. The working electrode is sealed with an O-ring, resulting in an effective area of $28 \mathrm{~mm}^{2}$ exposed to the liquid. The counter electrode is a gold wire (diameter $500 \mu \mathrm{m}$, oriented perpendicular to the flow direction) at a distance of $1.7 \mathrm{~mm}$ from the surface of the working electrode. The working electrode is pressed on a copper lid, serving as back electrode and heat sink together. Miniaturized thermocouples are integrated in the copper lid and in the liquid. The cell is equipped with a quartz glass bottom, enabling simultaneous fluorescence imaging with an inverted confocal fluorescence microscope. The syringe system comprises two identical programmable syringe pumps (ProSense, model NE-500, The Netherlands) enabling flow rates of $0.73 \mu \mathrm{l} \mathrm{h}^{-1}$ to $1699 \mathrm{ml} \mathrm{h}^{-1}$. One syringe serves for administering $0.1 \mathrm{M} \mathrm{NaOH}$ solution, the other delivers $1 \times$ phosphate buffered saline (PBS) solution. Both are connected to a computer-controlled three-way valve. The impedance spectroscopy unit measures the impedance in a frequency range of $100 \mathrm{~Hz}$ to $100 \mathrm{kHz}$ built up logarithmically with 10 frequencies per decade and a scanning speed of $5.7 \mathrm{~s}$ per sweep. All data discussed below refer to a frequency of 10 $\mathrm{kHz}$, ensuring an optimal signal-to-noise ratio. The amplitude of the $\mathrm{AC}$ voltage was fixed to $10 \mathrm{mV}$. For Nyquist plots covering the full frequency range, we refer to the ESI $\dagger$.

\section{Preparation of diamond-based sensor electrodes}

Nanocrystalline diamond (NCD) electrodes were selected for their intrinsic biocompatibility. ${ }^{23,24}$ Moreover, they allow for the covalent immobilization of biochemical receptors by various techniques. Examples are the binding of proteins ${ }^{25}$ and DNA fragments, ${ }^{26,27}$ where a manifold of at least 30 denaturationrehybridization cycles have been demonstrated without loss of

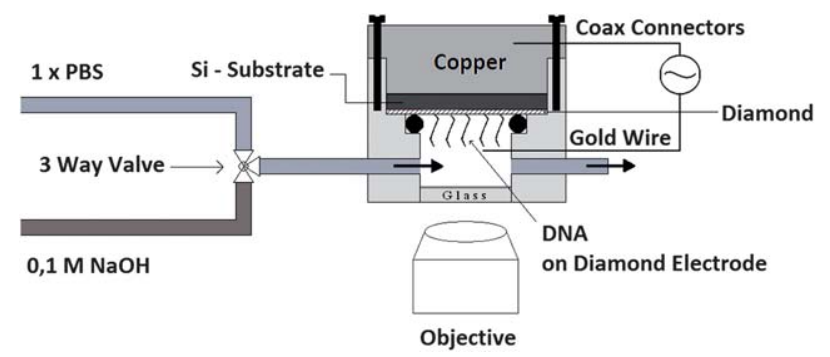

Fig. 1 Schematic layout of the impedimetric flow cell. The working electrode (DNA on nanocrystalline diamond on highly doped silicon) can be monitored with the fluorescence microscope while a gold wire serves as a counter electrode. The liquids $(1 \times$ PBS buffer and $0.1 \mathrm{M} \mathrm{NaOH})$ are sequentially administered by a syringe-driven pump system connected to a 3-way valve. The temperature of the liquid in the cell and of the copper back contact is measured by thermocouples (not shown). All connections to the impedance analyzer are done by mini-coax cables. 
binding capacity. An overview of functionalization strategies, both on oxygen- and on hydrogen-terminated diamond surfaces, can be found in the review articles by Wenmackers et l. $^{28}$ and Vermeeren et $a l .{ }^{29}$ Furthermore, diamond offers a wide electrochemical window and it is chemically and physically stable at elevated temperatures, under extreme $\mathrm{pH}$ conditions, and in solutions with high ionic strengths. These elements make NCD a universal electrode material for monitoring biochemical reactions in real time. DNA hybridization sensors based on diamond as a platform material have been reported in combination with impedance spectroscopy, ${ }^{21,30}$ solution-gate FETs, ${ }^{31}$ and cyclic voltammetry with a redox mediator on diamond nanowires. ${ }^{32}$ Here, planar sensor electrodes were prepared by microwave plasma-enhanced chemical vapour deposition (MW PE-CVD) from methane/hydrogen mixtures in an ASTeX reactor as described in ref. 33. The substrates were 2-inch silicon wafers (thickness 500-550 $\mu \mathrm{m}$, crystalline orientation (100), p-type doped with boron and resistivities from 1 to $20 \Omega \mathrm{cm}$ ), which were diced into samples of $10 \mathrm{~mm}$ by $10 \mathrm{~mm}$ after deposition. The diamond layers had a typical thickness of $100 \mathrm{~nm}$ with an average grain size of $50 \mathrm{~nm}$ as determined by X-ray diffraction and atomic force microscopy. Due to the nanocrystalline character of the diamond coating, no preferential crystallographic orientation was detectable. To ensure a good electrical conductivity of the diamond layer (in the order of $1 \Omega \mathrm{cm}$ ), the CVD deposition was done with an admixture of trimethyl borane $\left(\mathrm{B}\left(\mathrm{CH}_{3}\right)_{3}\right)$ to the $\mathrm{CH}_{4}$ gas with a concentration ratio of $200 \mathrm{ppm} \mathrm{B/C}$. The asprepared diamond electrodes were hydrogenated in $\mathrm{H}_{2}$ plasma (50 Torr, $800{ }^{\circ} \mathrm{C}$, power $4000 \mathrm{~W}$, duration of $2 \mathrm{~min}$ ) to facilitate the attachment of the fatty acid linker molecules as described in the next paragraph. In total, 5 different diamond electrodes have been studied, denoted as \# D1 to \# D5.

\section{Tethering of the probe DNA}

First, 10-undecenoic fatty acid was photochemically attached to the H-terminated NCD electrodes by UV illumination (wavelength $254 \mathrm{~nm}$, intensity $265 \mathrm{~mW} \mathrm{~cm}^{-2}$ ) for 20 hours under a protective $\mathrm{N}_{2}$ atmosphere. The reaction mechanism is presumably based on the fact that the hydrophobic $\mathrm{C}=\mathrm{C}$ end of the fatty acid is oriented towards the $\mathrm{H}$-terminated diamond surface and mediated by photoemission from the surface as proposed for the photochemical grafting of alkenes to silicon surfaces. ${ }^{34}$ After this photochemical treatment, the samples were thoroughly rinsed in acetic acid at $100{ }^{\circ} \mathrm{C}$ to remove unbound fatty acid fragments. In a second step, $\mathrm{NH}_{2}$-modified ssDNA (36-mer with the sequence $5^{\prime}-\mathrm{NH}_{2}-\mathrm{C}_{6} \mathrm{H}_{12}$ AAA AAA ACC CCT
GCA GCC CAT GTA TAC CCC CGA ACC-3') was covalently linked to the $\mathrm{COOH}$ group of the fatty acid using carbodiimide coupling. The details of this procedure and the final washing steps to remove non-reacted probe DNA have been described elsewhere. ${ }^{27,35}$ The origin and composition of chemical agents and buffer solutions are given in the ESI $\dagger$. The first 7 adenine bases at the $5^{\prime}$ terminus of the probe DNA serve as a spacer to avoid border effects at the proximity of the electrode surface. The total amount of probe ssDNA used to functionalize $1 \mathrm{~cm}^{2}$ of the electrode surface was 300 pmol. This is in excess of the binding capacity of the surface, yielding a rapid functionalization.

\section{Hybridization with target DNA}

Hybridization of the probe ssDNA molecules (36 bp) attached to the NCD was performed by incubating ssDNA-modified NCD samples for 2 hours at $30{ }^{\circ} \mathrm{C}$ with 600 pmol of Alexa 488-labelled target ssDNA ( $29 \mathrm{bp}$ ) in $10 \times$ polymerase chain reaction $(\mathrm{PCR})$ buffer. Four different types of target DNA have been employed: a sequence, which was complementary to the probe ssDNA, a random sequence, and two sequences with a 1-base mismatch at base pair 7 or base pair 20 respectively as shown in Table 1. Note that for both 1-base mismatch sequences the mismatch is a ' $\mathrm{CC}$ ' while the nearest neighbours are 'GC' and 'AT' in both cases. During hybridization, the samples were placed in a closed container under a saturated water vapour atmosphere to avoid evaporation of the reaction fluid. After hybridization, the samples were rinsed in $2 \times$ Saline Sodium Citrate (SSC) buffer containing $0.5 \%$ sodium dodecyl sulfate (SDS) for $30 \mathrm{~min}$ at room temperature, followed by two 5 min rinsing steps in $0.2 \times$ SSC buffer, once at $5{ }^{\circ} \mathrm{C}$ below the hybridization temperature and once at room temperature. From previous studies based on fluorescence intensity and spectroscopic UV ellipsometry, it is known that the areal density of immobilized DNA duplexes is $10^{12}$ molecules per $\mathrm{cm}^{2}$ and their typical tilt angle with respect to the normal of the electrode surface is approximately $50^{\circ} .^{35,36}$ The areal density corresponds to an average distance of $10 \mathrm{~nm}$ between neighbouring DNA strands, which is slightly less than the length of the probe DNA fragments (36 bases correspond to $12 \mathrm{~nm}$ ), but still corresponding to a diluted molecular brush without sterical hindering.

\section{Confocal fluorescence microscopy}

The denaturation of DNA was evaluated by measuring the change in fluorescence intensity with a Zeiss LSM 510 META Axiovert $200 \mathrm{M}$ laser scanning confocal fluorescence microscope

Table 1 Compilation of the base sequences of the probe DNA and the four different types of target DNA employed in the hybridization and denaturation experiments. The probe DNA exhibits a spacer consisting of 7 A-bases while the target DNA fragments carry a fluorescent Alexa 488 label at the $5^{\prime}$ end. The position of the mismatches with respect to the probe DNA is underlined and indicated by bold letters. $\alpha$ is the spacer between surface and the DNA strand being AAA AAA $\mathrm{C}_{6} \mathrm{H}_{12}-\mathrm{NH}_{2}-5^{\prime}$

\begin{tabular}{|c|c|}
\hline Name & Sequence \\
\hline Full match & $5^{\prime}$-Alexa $488-\mathrm{C}_{6} \mathrm{H}_{12}$ GGT TCG GGG GTA TAC ATG GGC TGC AGG GG-3' \\
\hline Mismatch at BP 20 & $5^{\prime}$-Alexa $488-\mathrm{C}_{6} \mathrm{H}_{12}$ GGT TCG GGG CTA TAC ATG GGC T $\overline{\mathrm{G} C}$ AGG GG-3' \\
\hline Random sequence & 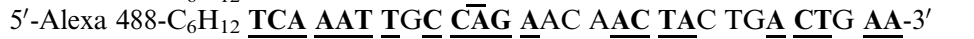 \\
\hline
\end{tabular}


using $488 \mathrm{~nm}$ argon-ion laser excitation with a maximum intensity at the sample surface of $1.00 \pm 0.05 \mathrm{~mW}$. All images were collected with a $10 \times / 0.3$ Plan Neofluar air objective with a working distance of $5.6 \mathrm{~mm}$. The image size was $128 \times 128$ with a pixel dwell time of $51.2 \mu \mathrm{s}$, corresponding to $\sim 900$ by $900 \mu \mathrm{m}^{2}$. The pinhole size was $150 \mu \mathrm{m}$ and the laser intensity was set at $10 \%$. The detector gain, being a measure for the photomultiplier voltage in arbitrary units, varied between 1000 and 1200 in different measurements. For photobleaching experiments, the laser intensity was set at $100 \%$ for 3 minutes. Fluorescence images were processed and the average fluorescence intensities were retrieved using the AIM4.2 software package. After hybridization with the different types of target DNA, each of the NCD samples was studied by confocal microscopy to ensure the presence and homogeneous distribution of DNA. Bleaching experiments demonstrated clearly that the fluorescence intensity originated from the Alexa 488 dyes and not from the underlying diamond layer.

\section{Results and discussion}

\section{C.1. Comparitive impedimetric and optical denaturation study}

A real-time denaturation experiment on perfectly matched dsDNA (electrode \# D1) is shown in Fig. 2. After mounting the functionalized electrode, the cell was filled with $1 \times$ PBS buffer and installed on the confocal fluorescence microscope. The cell was allowed to stabilize for $45 \mathrm{~min}$ to guarantee that drift effects were absent and the noise level was below $0.5 \%$. The moderate noise level in this measurement is related to the fact that the ambient temperature of about $25{ }^{\circ} \mathrm{C}$ could not be actively

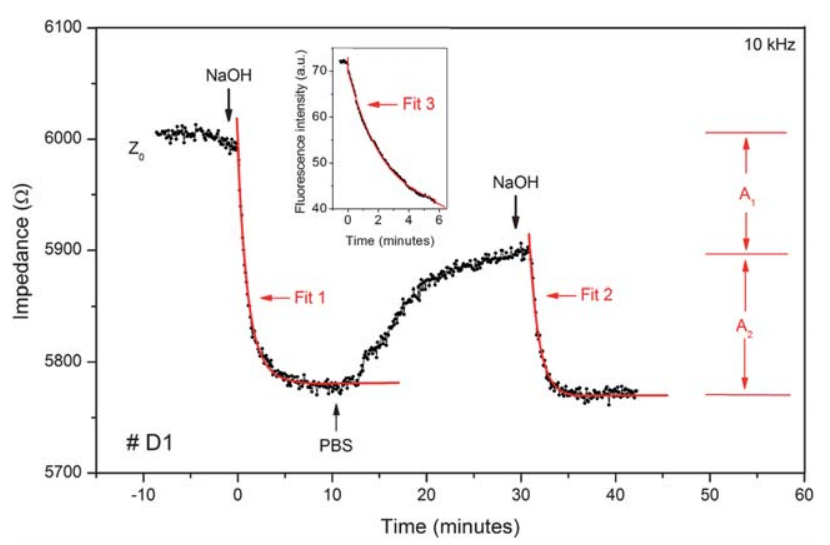

Fig. 2 Impedance profile of the denaturation of full-match DNA on diamond sample \# D1 with the time dependence of the fluorescence intensity in the inset. At $t=0,0.1 \mathrm{M} \mathrm{NaOH}$ enters the cell and replaces the $1 \times$ PBS buffer, causing a drop of the fluorescence intensity and the impedance. This emerges from the denaturation and the change of liquids, while the fluorescence drop stems from DNA denaturation only. The extraction of the time constants $\tau_{1}$ (denaturation) and $\tau_{2}$ (exchange of fluids) is based on the Fits 1 and 2, as described in the text. The time constant $\tau_{1}=2.24 \pm 0.14$ min from the impedimetric data is consistent with the fluorimetric result $\tau_{3} \approx 2.41 \mathrm{~min}$ obtained from Fit 3 . To determine $\tau_{2}=0.97 \pm 0.06 \mathrm{~min}$, the cell was refilled with $1 \times$ PBS at $t_{1}=12 \mathrm{~min}$ and flushed with $0.1 \mathrm{M} \mathrm{NaOH}$ at $t_{2}=30 \mathrm{~min}$. The amplitudes $A_{1}$ and $A_{2}$ indicate the respective influence of denaturation and liquids exchange on the impedance changes. controlled. In all further measurements, not performed on the inverted confocal microscope, the ambient temperature and temperature of all liquids were strictly stabilized to $19.3{ }^{\circ} \mathrm{C}$, resulting in even lower noise levels. At $t_{0}=0 \mathrm{~min}, 0.1 \mathrm{M} \mathrm{NaOH}$ at a flow rate of $250 \mu \mathrm{l}$ per minute enters the cell and replaces the PBS filling. This results in an impedance drop, which consists of two separate contributions: (i) the intrinsic effect of denaturation, which affects the electronic properties in the vicinity of the topmost diamond layer and (ii) the medium exchange as the 0.1 $\mathrm{M} \mathrm{NaOH}$ filling causes a higher conductivity than $1 \times$ PBS. To distinguish both contributions quantitatively, the $0.1 \mathrm{M} \mathrm{NaOH}$ was replaced by reintroducing $1 \times$ PBS at $t_{1}=12 \mathrm{~min}$ (flow rate $250 \mu 1 \mathrm{~min}^{-1}$ ). The impedance increases and stabilizes at a plateau with a lower value as compared to the starting condition. This demonstrates that the impedimetric properties of the electrode surface must have changed upon denaturation because the ionic properties of the PBS buffer are identical before and after the inbetween denaturation step. To analyze the typical timescale of introducing $0.1 \mathrm{M} \mathrm{NaOH}$, at $t_{2}=30 \mathrm{~min}$ the $1 \times \mathrm{PBS}$ was finally replaced by $0.1 \mathrm{M} \mathrm{NaOH}$ at a flow rate of $250 \mu 1 \mathrm{~min}^{-1}$. The superimposed processes of denaturation and the pure effect of medium exchange can mathematically be described as follows:

$$
\begin{gathered}
Z(t)=Z(t=\infty)+A_{1} \exp \left\{-\frac{t}{\tau_{1}}\right\}+A_{2} \exp \left\{-\frac{t}{\tau_{2}}\right\} \\
Z(t)=Z(t=\infty)+A_{2} \exp \left\{-\frac{t}{\tau_{2}}\right\}
\end{gathered}
$$

The double-exponential fit according to eqn (1) for superimposed, independent decay processes is known e.g., from the decomposition of biomass (tomato leaves) and the mass loss of tomato DNA as a function of time. ${ }^{37}$ The parameter $A_{1}$ represents the denaturation-related decay amplitude and $\tau_{1}$ the associated time constant; the amplitude $A_{2}$ refers to the impedance drop by the medium exchange and $\tau_{2}$ is the corresponding time constant. The fit according to eqn (2) describes solely the influence of the medium exchange from $1 \times$ PBS to $0.1 \mathrm{M} \mathrm{NaOH}$ after the denaturation has taken place and is therefore representative for the medium exchange as such. Note that there is no intrinsic reason for the exponential time dependence related to the medium exchange, but the agreement with experimental data is excellent with a coefficient of determination $\left(R^{2}\right)$ of 0.97 for the fit with eqn (2). The exponential time dependence of the splitting of DNA duplexes is naturally inherent to decay processes of noninteracting ensembles. First, we applied the fit according to eqn (2) and extracted $\tau_{2}=0.97 \pm 0.06$ min together with $A_{2}=112 \pm 9$ $\Omega$ for the medium exchange effect. Inserting these values into the fit based on eqn (1) resulted in a denaturation-time constant $\tau_{1}=2.24 \pm 0.14 \mathrm{~min}$ and an amplitude $A_{1}=115 \pm 13 \Omega$. The $R^{2}$ of the fit according to eqn (1) is 0.94 , giving support to the concept of superimposed decay processes. All fits are performed with Origin 7.1. To cross-check the electronically determined $\tau_{1}$, time-lapse fluorescence imaging was performed during the denaturation step (time interval of 1.4 seconds between subsequent images) and the intensity $I(t)$ was averaged over an area of 900 by $900 \mu \mathrm{m}^{2}$. Selected images with intervals of 36 seconds, taken during the first 6 minutes of the dynamic imaging, are 


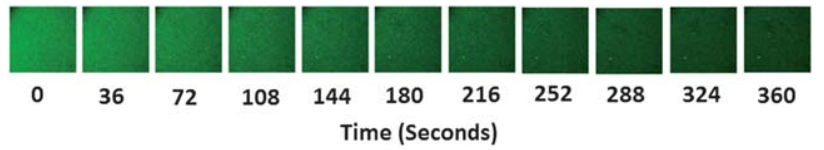

Fig. 3 Series of confocal fluorescence images of the diamond electrode during the denaturation of perfectly complementary dsDNA at selected times. Note that the fluorescence-intensity curve in Fig. 2 was derived from images with time intervals of 1.4 seconds. The depicted area of each square is 900 by $900 \mu \mathrm{m}^{2}$.

shown in Fig. 3. We note that there is a remnant background intensity $I_{0}$, which is not vanishing even long after this period, and therefore attributed to the reflected laser light. The areaaveraged intensity values are shown as an inset in Fig. 2 and described with the fit according to eqn (3):

$$
I(t)=I_{0}+I_{\mathrm{DNA}} \exp \left\{-\frac{t}{\tau_{3}}\right\}
$$

The fluorescence decay time constant $\tau_{3}=2.41 \pm 0.05 \mathrm{~min}$ is, within the error margins, perfectly consistent with the electronically determined $\tau_{1}$. The determination of $\tau_{3}$ is insensitive to the medium composition and truly reflects the progressing denaturation at the NCD electrode surface. The Alexa 488 labeled target DNA fragments, which are removed by $\mathrm{NaOH}$ exposure, do not contribute to the fluorescence intensity as they are transported away by the constant $\mathrm{NaOH}$ flow while the confocal volume is restricted to a distance of less than $4.5 \mu \mathrm{m}$ from the surface of the NCD electrode. In conclusion, the electronically determined denaturation time constant $\tau_{1}$ is a reliable measure for the duration of the chemically induced denaturation process. It is important for the applicability of the approach that the time constant of the medium exchange is shorter than the duration of the denaturation process. In the opposite case (denaturation faster than medium exchange), the medium exchange would be the determining factor for the progressing DNA denaturation, making it more difficult to determine the time constant of the denaturation process. This is a useful aspect in the sense that the medium exchange time constant can be adjusted in wide ranges by adapting the flow rate of the pumping system to meet conditions in which the medium exchange is considerably faster than the dynamics of biological recognition or unbinding events. Repeating the entire procedure on diamond sample \# D1, rehybridized with complementary target DNA fragments, but measured in a temperature-stabilized environment of $19.3^{\circ} \mathrm{C}$ and without laser illumination by the confocal microscope, gave $\tau_{1}=$ $2.28 \pm 0.16 \mathrm{~min}$ and $\tau_{2}=0.58 \pm 0.04 \mathrm{~min}$. The denaturation time constant is clearly consistent with the measurement at $25{ }^{\circ} \mathrm{C}$ although the influence of the medium exchange levels off faster, due to a slight modification to the flow system. In a next step, the probe DNA was hybridized with target DNA with a single nucleotide mismatch at base pair 7. This resulted in $\tau_{1}=1.21 \pm$ $0.10 \mathrm{~min}$ and $\tau_{2}=0.48 \pm 0.02 \mathrm{~min}$. As expected, the time constant for the medium exchange is very similar under identical environmental and flow conditions, but the DNA duplexes with the SNP mutation denature considerably faster than the complementary duplexes. This strongly suggests that the monitoring of chemically induced denaturation can give an indication for the presence of single nucleotide polymorphisms.
C.2. Impedimetric denaturation monitoring with various types of target DNA

In order to evaluate the reproducibility of the method and whether in principle it allows to localize and to identify SNPs, we performed systematic studies on four additional diamond electrodes (\# D2, \# D3, \# D4, and \# D5). Each DNA-modified electrode was hybridized with four different types of target DNA: the perfect complement, the $\mathrm{CC}$ mismatch at base pair 7, the $\mathrm{CC}$ mismatch at base pair 20 (further away from the diamond electrode and closer to the distal end of the molecular brush), and a random-type target DNA. The random target can also form transient Watson-Crick pairs but this is limited to a few nucleotides as shown in Table 1. Note that during the consecutive hybridizations with the different variants of target DNA, always the initial probe DNA has been used without any regeneration treatment. The results obtained with electrode \# D2 are shown in Fig. 4. All measurements on \# D2 (and on \# D3, \# D4, and \# D5) were also performed under temperature-stabilized conditions, which resulted in excellent $R^{2}$ values between 0.96 and 0.996 for the Fits 1 and 2. In Fig. 4, the first impedance decay (additive effect of DNA denaturation and medium exchange) is normalized to the impedance at $t=0 \mathrm{~min}$; the second impedance decay is normalized to the impedance value at the time $t_{2}$, when the bound DNA has already been denatured and $1 \times$ PBS is again replaced by $0.1 \mathrm{M} \mathrm{NaOH}$. All data on the denaturation time constant $\tau_{1}$, obtained at $19.3{ }^{\circ} \mathrm{C}$ with the five different electrodes, are summarized in Fig. 5. The time constants $\tau_{2}$ for the medium exchange are depicted for comparison. The averaged time constants for denaturation and medium exchange and the averaged normalized amplitudes are summarized in Table 2 together with their respective standard deviation $\sigma$. Concerning $\left\langle\tau_{1}\right\rangle$, we obtain $2.26 \pm 0.11 \mathrm{~min}$ for complementary duplexes, $1.38 \pm 0.05$ min for the mismatch at base pair 20, $1.16 \pm 0.04 \mathrm{~min}$ for the mismatch at base pair 7, and finally $0.59 \pm 0.08 \mathrm{~min}$ for the random target. The time constant for denaturation of the random sequence is close to $\left\langle\tau_{2}\right\rangle=0.46 \pm 0.04 \mathrm{~min}$ for the medium exchange, indicating that these fragments are at the most very loosely bound. To ensure that the marked difference in time constants is not emerging from an electrode-aging effect during consecutive denaturation processes and exposure to 0.1 $\mathrm{M} \mathrm{NaOH}$, the order of hybridization and denaturation with the different types of target DNA was also considered. The samples \# D2, \# D3, and \# D4 were first hybridized with the complementary sequence, second with the mismatch at base pair 7 , third with the mismatch at base pair 20, and finally with the random sequence. In the case of sample \# D5 the order was reversed, starting with the random sequence. As a result, all time constants determined with \# D5 were found to be fully in line with the other electrodes.

Furthermore, we studied also the intra-sample reproducibility by hybridizing and denaturating electrode \# D1 six times with the complementary target-DNA sequence for a period of more than 4 months. The results are summarized in Table 3. The averaged value of the denaturation-time constant $\left\langle\tau_{1}\right\rangle$ is $2.25 \mathrm{~min}$ with a standard deviation $\sigma\left\langle\tau_{1}\right\rangle=0.18 \mathrm{~min}$. This is comparable to the data obtained as an average over the same measurement (denaturation of complementary duplexes) performed with the five different electrodes \# D1 to \# D5. There, we obtained 

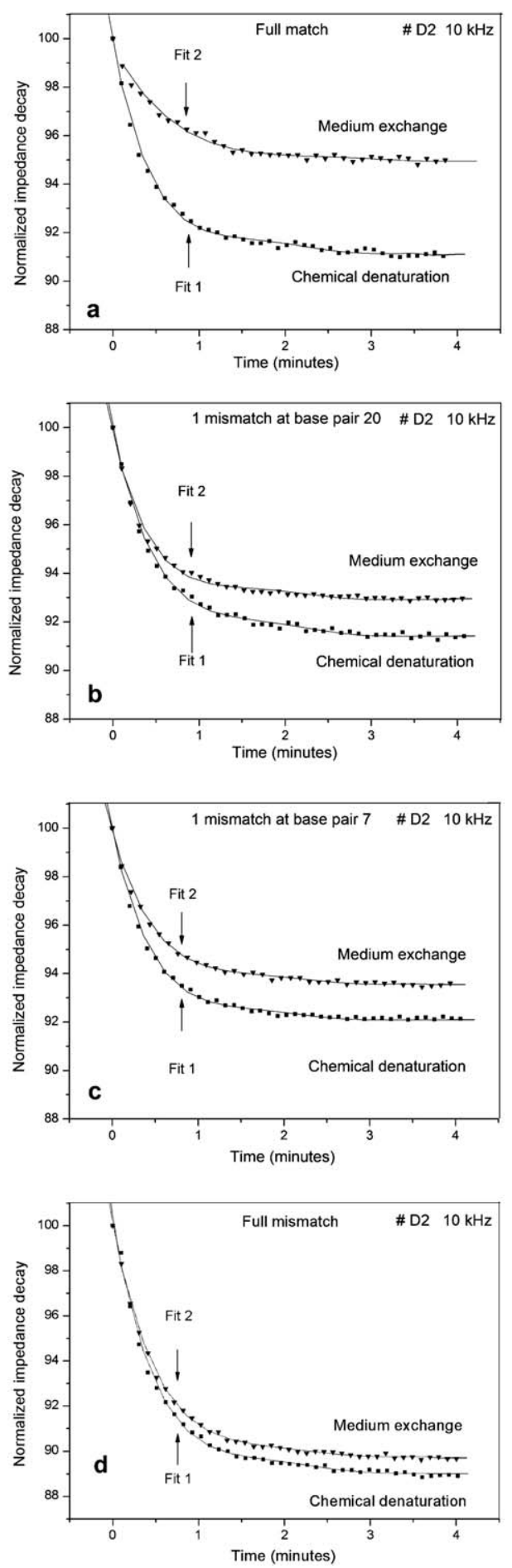

Fig. 4 Overview of the denaturation steps performed on diamond sample \# D2 with complementary target DNA (a), mismatch at base pair 20 (b), mismatch at base pair 7 (c), and the random target sequence (d). All data described as 'chemical denaturation' are normalized to the impedance value at the moment when $0.1 \mathrm{M} \mathrm{NaOH}$ enters the cell and fitted with the double exponential eqn (1) (Fit 1) as described in the text. The curves denoted as 'medium exchange' refer to the second replacement of $1 \times$ PBS by $0.1 \mathrm{M} \mathrm{NaOH}$ after the actual denaturation step. These data are normalized with respect to the equilibrium impedance value at the time $t_{2}$ obtained after refilling the cell with $1 \times$ PBS buffer (Fit 2 according to eqn (2)).

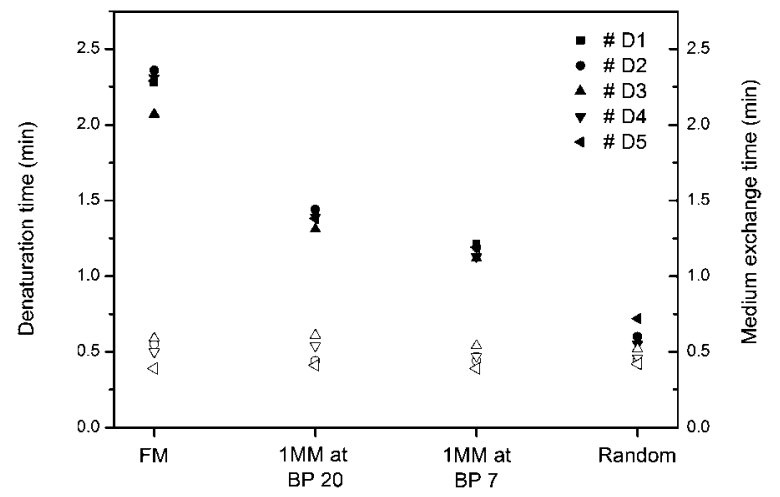

Fig. 5 Compilation of the denaturation-time constants $\tau_{1}$ (solid symbols) for the four different types of target DNA. Each time constant was measured on at least four different diamond electrodes and the scattering between the data is remarkably low. The time constants $\tau_{2}$ for medium exchange from $1 \times$ PBS to $0.1 \mathrm{M} \mathrm{NaOH}$ are indicated by open symbols. Note that even in the case of the random sequence there is still a slight difference between the denaturation time and the medium exchange.

$\left\langle\tau_{1}\right\rangle=2.26$ min with a standard deviation $\sigma\left\langle\tau_{1}\right\rangle=0.11 \mathrm{~min}$ as given in Table 2 . The averaged values of this intra-versus interelectrode comparison are practically identical. The slightly wider data scattering for the intra-electrode measurements can be attributed to the elongated time span in between these measurements with a possible influence on the biochemical reagents. The averaged time constant for the medium exchange $\left\langle\tau_{2}\right\rangle$ and the corresponding standard deviation $\sigma\left\langle\tau_{2}\right\rangle$ agree again well with the data in Table 2 obtained with the five different electrodes.

Since the melting temperature $T_{\mathrm{m}}$ is the established measure for the stability of DNA duplexes and the key parameter in localization and identification of single nucleotide polymorphisms, we employed two different algorithms to estimate $T_{\mathrm{m}}$ for the four different target-probe duplexes in our study. FractTM is available online (http://www.zaik.uni-koeln.de/ bioinformatik/fptm.html) and the underlying principles are described in ref. 38. HYTHER ${ }^{\mathrm{TM}}$ is also available online and allows taking into account that the $5^{\prime}$ end of the probe DNA is tethered to a solid support (http://ozone3.chem.wayne.edu/). Both algorithms calculate $T_{\mathrm{m}}$ on the basis of the sequence of probe and target DNA, nearest neighbor effects, the concentration of the probe DNA, and the $\mathrm{Na}^{+}$concentration of the surrounding electrolyte. The numerical results of both algorithms are summarized in Table 2. Despite minor differences in the absolute $T_{\mathrm{m}}$ values, the global trend is in agreement with the obtained denaturation time constants: the complementary duplexes have the highest melting temperature and the longest denaturation time, the duplexes with the random sequence have the lowest $T_{\mathrm{m}}$ and shortest $\tau_{1}$. Negative $T_{\mathrm{m}}$ values in the case of the random sequence mean that these duplexes are unstable at any positive temperature because the calculations do not take into account the freezing of the aqueous matrix. The melting temperature of the SNP duplexes is reduced by 3 to $6{ }^{\circ} \mathrm{C}$ as compared to their fully complementary counterparts while we observe here a strongly decreased denaturation time. Interestingly enough, both algorithms predict a slightly higher stability 
Table 2 Comparison of the theoretical melting temperatures with the parameters deduced from the real-time denaturation experiments. The melting temperatures were calculated using the FractTM algorithm (values for filter hybrization in brackets) (http://www.zaik.uni-koeln.de/bioinformatik/fptm. html, ref.38) and the HYTHER ${ }^{\mathrm{TM}}$ algorithm (http://ozone3.chem.wayne.edu). The denaturation-time constant $\left\langle\tau_{1}\right\rangle$, the time constant of the medium exchange $\left\langle\tau_{2}\right\rangle$, the normalized amplitude $\left\langle A_{1} / Z(0)\right\rangle$ related to DNA denaturation, and the relative amplitude $\left\langle A_{2} / Z\left(t_{2}\right)\right\rangle$ related to the medium exchange are averages of four to five independent measurements. The $\sigma$ values are the respective standard deviations. Note that the $\left\langle\tau_{2}\right\rangle$ values are almost indistinguishable and that $\left\langle A_{1} / Z(0)\right\rangle$ can be considered as a measure of the quantity of bound target DNA

\begin{tabular}{|c|c|c|c|c|}
\hline Target DNA & Complement & Mismatch BP 20 & Mismatch BP 7 & Random \\
\hline$T$ melting $/{ }^{\circ} \mathrm{C}$ (HYTHER) & 79.5 & 75.0 & 76.7 & -50.8 \\
\hline$\sigma\left\langle\tau_{1}\right\rangle / \min$ & 0.11 & 0.05 & 0.04 & 0.08 \\
\hline$\left\langle\tau_{2}\right\rangle / \mathrm{min}$ & 0.52 & 0.50 & 0.46 & 0.46 \\
\hline$\sigma\left\langle\tau_{2}\right\rangle / \min$ & 0.08 & 0.09 & 0.06 & 0.04 \\
\hline$\left\langle A_{1} / Z(0)\right\rangle(\%)$ & 3.4 & 2.0 & 2.0 & 0.4 \\
\hline$\sigma\left\langle A_{2} / Z\left(t_{2}\right)\right\rangle(\%)$ & 1.3 & 0.5 & 1.2 & 1.1 \\
\hline
\end{tabular}

for the duplex with the SNP at base pair 7 (HYTHER: $T_{\mathrm{m}}=76.7$ ${ }^{\circ} \mathrm{C}$ ) compared to the SNP at base pair 20 (HYTHER: $T_{\mathrm{m}}=75.0$ $\left.{ }^{\circ} \mathrm{C}\right)$. This difference is minimal, note that the type of the defect and its nearest neighbors are identical, but our data suggest that the duplexes with the SNP at base pair 7 denature slightly faster than those with the SNP at base pair 20. The average denaturation time for the SNP at base pair 20 is $1.38 \pm 0.05 \mathrm{~min}$, while this is reduced to $1.16 \pm 0.04$ min for the SNP position at base pair 7. Within our statistics of four to five independent measurements per defect position, there is no overlapping of error bars and the method seems to allow distinguishing between both positions. Further studies will be necessary to clarify whether this deviation from thermodynamics-based $T_{\mathrm{m}}$ calculations is significant and to understand the kinetics of chemically induced denaturation close to a sensor electrode at the level of individual DNA molecules.

Finally, also the amplitude parameter $A_{1}$ gives information on the amount of bound target DNA. There is a systematic decrease of the normalized value $A_{1} / Z_{0}$ from $3.4 \%$ (complementary target), to $2.0 \%$ (mismatch at base pair 20 or at base pair 7) and finally to $0.3 \%$ for the random sequence, which is close to a zero effect within the standard deviation. Keeping in mind that the hybridization was performed under standardized conditions (see Section B) it is evident that defected sequences bind to a lesser extent than the complementary fragments,

Table 3 Time constants for denaturation $\left(\tau_{1}\right)$ and medium exchange $\left(\tau_{2}\right)$ for sample \# D1, hybridized with the complementary target DNA. The sample passed six hybridization-denaturation cycles over a period of 132 days. Note that all decay times, including the average values and standard deviations, are in close agreement with the results depicted in Table 2. This indicates a high inter- and intra-sample reproducibility

\begin{tabular}{lll}
\hline$\# \mathrm{D} 1$ & $\tau_{1} / \mathrm{min}$ & $\tau_{2} / \mathrm{min}$ \\
\hline Day 1 & $2.39 \pm 0.08$ & $0.49 \pm 0.13$ \\
Day 44 & $2.21 \pm 0.14$ & $0.50 \pm 0.07$ \\
Day 49 & $2.10 \pm 0.16$ & $0.55 \pm 0.09$ \\
Day 50 & $2.02 \pm 0.04$ & $0.45 \pm 0.08$ \\
Day 84 & $2.51 \pm 0.02$ & $0.46 \pm 0.15$ \\
Day 132 & $2.28 \pm 0.16$ & $0.58 \pm 0.04$ \\
$\left\langle\tau_{1,2}\right\rangle$ & 2.25 & 0.50 \\
$\sigma\left\langle\tau_{1,2}\right\rangle$ & 0.18 & 0.05 \\
\hline
\end{tabular}

which result in duplexes with the highest thermodynamic stability. Except for the random sequence, the normalized $A_{2} / Z\left(t_{2}\right)$ is identical for all samples and sequences within the error margins.

\section{Conclusions}

In summary, we have used impedance spectroscopy in combination with diamond-based sensor electrodes to monitor the kinetics of chemically induced DNA denaturation in real-time. The probe DNA was covalently immobilized onto the electrodes and for four different types of target DNA (complementary, random, single mismatches at two different positions) we could reproducibly identify denaturation time constants. All time constants are at the scale of minutes while the denaturation takes the longest for complementary DNA duplexes. Moreover, the time constants correlate well with calculated melting temperatures. This suggests that the novel approach may possibly allow for a fast localization and identification of SNPs similar to the established, but cumbersome DNA melting techniques. In addition, the novel method is intrinsically label-free (note that labels were only used to allow for fluorescence imaging as a reference technique) and as-prepared electrodes can be used repetitively, thanks to the strong covalent link between probe DNA and the diamond platform and the chemical inertness of diamond as such. The required instrumentation consists of an impedance analyzer, which can simply operate at a fixed frequency, and a system to administer $\mathrm{NaOH}$ with defined temperature and flow rate. With practical applications in mind, we point out that it is not essential to do the full fitting and analyses of the impedance decay curves. Any indication for the duration of the denaturation process like a response time criterium ( $95 \%$ of signal change) will presumably correlate with melting temperature data. Finally, the proposed method has the potential to be downsized and parallelized towards a microarraylike format as illustrated by the diamond-based microelectrode arrays with spot sizes of just $50 \mu \mathrm{m}$ as described in ref. 39. Given the fact that the real-time denaturation method provides 'dynamic information', it can reduce the number of required spots considerably as compared to the 'endpoint character' of classical microarrays with fluorimetric readout. 


\section{Acknowledgements}

This work was supported by FWO-Research Foundation Flanders (project G.0829.09—Synthetic diamond films as platform materials for novel DNA sensors based on electronic detection techniques), by the Belgian Interuniversity Attraction Pole Programme IUAP VI (Quantum effects in clusters and nanowires), by the Special Research Funds BOF of Hasselt University, and by the Life-Sciences Impulse Programme of the Belgian Province of Limburg. L. Grieten and V. Vermeeren are both supported by IWT - Agency for Innovation by Science and Technology. The authors are very grateful for technical support by Andreas Gaulke (University of Applied Sciences Kaiserslautern), Johnny Baccus, Lieven De Winter, Jan Mertens, and Johan Soogen (all in Hasselt University). Furthermore, stimulating scientific discussions with Prof. Jef Hooyberghs (Flemish Institute for Technological Research VITO, Mol/Belgium) and Dr Anitha Ethirajan and Prof. Hans-Gerd Boyen (both in Hasselt University) are greatly appreciated.

\section{References}

1 P. Y. Kwok, Annu. Rev. Genomics Hum. Genet., 2001, 2, 235-258.

2 J. K. K. Ng and W.-T. Liu, Anal. Bioanal. Chem., 2006, 3, 427434.

3 J. Hooyberghs and E. Carlon, Biosens. Bioelectron., 2010, 26, 16921695.

4 E. A. Tindall, D. C. Petersen, P. Woodbridge, K. Schipany and V. M. Hayes, Hum. Mutat., 2009, 30, 876-883.

5 R. Fodde and M. Losekoot, Hum. Mutat., 1994, 3, 83-94.

6 L. Lodewyckx, C. Vandevyver, C. Vandervorst, W. Van Steenbergen, J. Raus and L. Michiels, Hum. Mutat., 2001, 18, 243-250.

7 C. Dekker, Nat. Nanotechnol., 2007, 2, 209-215.

8 Y. Astier, O. Braha and H. Bayley, J. Am. Chem. Soc., 2006, 128, 10684-10685.

9 J. Clarke, H. C. Wu, L. Jayasinghe, A. Patel, S. Reid and H. Bayley, Nat. Nanotechnol., 2009, 4, 265-270.

10 U. Rant, K. Arinaga, S. Scherer, E. Pringsheim, S. Fujita, N. Yokoyama, M. Tornow and G. Abstreiter, Proc. Natl. Acad. Sci. U. S. A., 2007, 104, 17364-17369.

11 Y. Xiao, A. A. Lubin, B. R. Baker, K. W. Plaxco and A. J. Heeger, Proc. Natl. Acad. Sci. U. S. A., 2006, 103, 4017-4021.

12 F. Uslu, S. Ingebrandt, D. Mayer, S. Böcker-Meffert, M. Odenthal and A. Offenhäusser, Biosens. Bioelectron., 2004, 19, 1721-1731.

13 T. Sakata and Y. Miyahara, ChemBioChem, 2005, 6, 703-710.

14 S. Ingebrandt, Y. Han, F. Nakamura, A. Poghossian, M. J. Schöning and A. Offenhäusser, Biosens. Bioelectron., 2007, 22, 2834-2840.

15 A. Poghossian, A. Cherstvy, S. Ingebrandt, A. Offenhäusser and M. J. Schöning, Sens. Actuators, B, 2005, 111, 470-480.

16 F. Davis, M. A. Hughes, A. R. Cossins and S. P. J. Higson, Anal. Chem., 2007, 79, 1153-1157.

17 J.-Y. Park and S.-M. Park, Sensors, 2009, 9, 9513-9532.
18 E. Özkumur, S. Ahn, A. Yalcin, C. A. Lopez, E. Cevik, R. J. Irani, C. DeLisi, M. Chiari and M. S. Unlü, Biosens. Bioelectron., 2010, 25, 1789-1795.

19 J. Hooyberghs, M. Baiesi, A. Ferrantini and E. Carlon, Phys. Rev. E: Stat., Nonlinear, Soft Matter Phys., 2010, 81, 012901.

20 E. Katz and I. Willner, Electroanalysis, 2003, 15, 913-947.

21 V. Vermeeren, N. Bijnens, S. Wenmackers, M. Daenen, K. Haenen, O. A. Williams, M. Ameloot, M. vandeVen, P. Wagner and L. Michiels, Langmuir, 2007, 23, 13193-13202.

22 B. van Grinsven, T. Vandenryt, S. Duchateau, A. Gaulke, L. Grieten, R. Thoelen, S. Ingebrandt, W. De Ceuninck and P. Wagner, Phys. Status Solidi A, 2010, 207, 2110-2113.

23 L. Tang, C. Tsai, W. W. Gerberich, L. Kruckeberg and D. R. Kania, Biomaterials, 1995, 16, 483-488.

24 N. Smisdom, I. Smets, O. A. Williams, M. Daenen, S. Wenmackers, K. Haenen, M. Nesládek, J. D'Haen, P. Wagner, J.-M. Rigo, M. Ameloot and M. vandeVen, Phys. Status Solidi A, 2009, 206, 2042-2046.

25 A. Härtl, E. Schmich, J. A. Garrido, J. Hernando, S. C. R. Catharino, S. Walter, P. Feulner, A. Kromka, D. Steinmüller and M. Stutzmann, Nat. Mater., 2004, 3, 736-742.

26 W. Yang, O. Auciello, J. E. Butler, W. Cai, J. A. Carlisle, J. E. Gerbi, D. M. Gruen, T. Knickerbocker, T. L. Lasseter, J. N. Russell, Jr, L. M. Smith and R. J. Hamers, Nat. Mater., 2002, 1, 253-257.

27 P. Christiaens, V. Vermeeren, S. Wenmackers, M. Daenen, K. Haenen, M. Nesládek, M. vandeVen, M. Ameloot, L. Michiels and P. Wagner, Biosens. Bioelectron., 2006, 22, 170-177.

28 S. Wenmackers, V. Vermeeren, M. vandeVen, M. Ameloot, N. Bijnens, K. Haenen, L. Michiels and P. Wagner, Phys. Status Solidi A, 2009, 206, 391-408.

29 V. Vermeeren, S. Wenmackers, P. Wagner and L. Michiels, Sensors, 2009, 9, 5600-5636.

30 H. Gu, X. Su and K. P. Loh, J. Phys. Chem. B, 2005, 109, 1361113618.

31 S. Kuga, S. Tajima, J. H. Yang, K. Hirama and H. Kawarada, IEEE Int. Electron Devices Meet., Tech. Dig., 50th, 2008, 483-486.

32 N. Yang, H. Uetsuka, E. Osawa and C. E. Nebel, Angew. Chem., Int. Ed., 2008, 47, 5183-5185.

33 O. A. Williams, M. Nesládek, M. Daenen, S. Michaelson, A. Hoffman, E. Osawa, K. Haenen and R. B. Jackman, Diamond Relat. Mater., 2008, 17, 1080-1088.

34 X. Y. Wang, R. E. Ruther, J. A. Streifer and R. J. Hamers, J. Am. Chem. Soc., 2010, 132, 4048-4049.

35 V. Vermeeren, S. Wenmackers, M. Daenen, K. Haenen, O. A. Williams, M. Ameloot, M. vandeVen, P. Wagner and L. Michiels, Langmuir, 2008, 24, 13193-13202.

36 S. Wenmackers, S. D. Pop, K. Roodenko, V. Vermeeren, O. A. Williams, M. Daenen, O. Douheret, J. D'Haen, A. Hardy, M. K. Van Bael, K. Hinrichs, C. Cobet, M. vandeVen, M. Ameloot, K. Haenen, L. Michiels, N. Esser and P. Wagner, Langmuir, 2008, 24, 7269-7299.

37 J. Poté, P. Rossé, W. Roselli, W. T. Van and W. Wildi, Chemosphere, 2005, 61, 677-684.

38 M. Leber, L. Kaderali, A. Schönhuth and R. Schrader, Bioinformatics, 2005, 21, 2375-2382.

39 M. Bonnauron, S. Saada, C. Mer, C. Gesset, O. A. Williams, L. Rousseau, E. Scorsone, P. Mailley, M. Nesládek, J. C. Arnault and P. Bergonzo, Phys. Status Solidi A, 2008, 205, 2126-2129. 